\title{
Holographic Dark Energy Model and Scalar-Tensor Theories
}

\author{
Yousef Bisabr* \\ Department of Physics, Shahid Rajaee University, Lavizan, Tehran 16788, Iran.
}

\begin{abstract}
We study the holographic dark energy model in a generalized scalar tensor theory. In a universe filled with cold dark matter and dark energy, the effect of potential of the scalar field is investigated in the equation of state parameter. We show that for a various types of potentials, the equation of state parameter is negative and transition from deceleration to acceleration expansion of the universe is possible.
\end{abstract}

\section{Introduction}

It is strongly believed that our universe is in a phase experiencing an accelerated expansion. The recent observations regarding the luminosity-redshift relation of type Ia supernovae [1] in association with observations on Cosmic Microwave Background Radiation [2] demonstrate this cosmic acceleration. The simplest candidate to produce this cosmic speed-up is the cosmological constant, the energy density associated with quantum vacuum. However, there are at least two problems for associating cosmic acceleration with the cosmological constant. Firstly, theoretical estimates on its value are many order of magnitude larger than observations [4]. Secondly, it is simply a constant, namely that it is not diluted with expansion of the universe. This latter is specifically important in the sense that there are observational evidence [5] demonstrating that the cosmic expansion is a recent phenomena and the universe must have passed through a deceleration phase in the early stages of its evolution. This deceleration phase is important for successful nucleosynthesis as well as for the structure formation. We therefore need a field evolving during expansion of the universe in such a way that its dynamics makes the deceleration parameter have a signature flip from positive in the early stages of

*e-mail: y-bisabr@srttu.edu. 
matter dominated era to negative in the present stage.

This fact has motivated people to consider many dynamical models trying to explain the cosmic acceleration. Among these models there are those that assumes a mysterious cosmic fluid with sufficiently large and negative pressure, dubbed dark energy. These models are usually invoked a scalar field which during its evolution takes negative pressure by rolling down a proper potential. There is a large class of scalar field models in the literature including, quintessence [6], k-essence [7], tachyons [8], phantom field [9], quintom [10], and so forth. However, most of these models are not compelling enough and require fine tuning of parameters to be consistent with observations.

There is another proposal, first formulated by 't Hooft and Susskind [11], which recently has attracted much attention as a possible solution to the dark energy problem. The basic idea, dubbed holographic principle, is that the number of degrees of freedom of a physical system scales with its bounding area rather than with its volume. For an effective quantum field theory in a box of size $L$ with an ultraviolet (UV) cutoff $\Lambda$, the entropy $S$ scales extensively as $S \sim L^{3} \Lambda^{3}$. However the peculiar thermodynamics of black holes has led Bekenstein [12] to postulate that the maximum entropy in a box of volume $L^{3}$ behaves non-extensively, growing as the area of the box. In this sense there is a so-called Bekenstein entropy bound

$$
S=L^{3} \Lambda^{3} \leq S_{B H} \equiv \pi L^{2} M_{p}^{2}
$$

where $S_{B H}$ is the entropy of a black hole of radius $L$, and $M_{p} \equiv(8 \pi G)^{-\frac{1}{2}}$ stands for the reduced Planck mass. It is important that in this relation the length scale $L$ providing an Infrared (IR) cutoff is determined by the UV cutoff $\Lambda$ and can not be chosen independently. However such a non-extensive scaling law seems to provide a breakdown of quantum field theory at large scales. To reconcile this breakdown with the success of local quantum field theory in describing observed particle phenomenology, Cohen et al. [13] proposed a more restrictive bound. Since the maximal energy density in the effective theory is of the order $\rho_{\Lambda}=\Lambda^{4}$, requiring that the energy in a given volume not to exceed the energy of a black hole of the same size results in the constraint

$$
L^{3} \rho_{\Lambda} \leq L M_{p}^{2}
$$

If we take the largest value of the length scale $L$ as the IR cutoff saturating the inequality (2), we then obtain the holographic dark energy density

$$
\rho_{\Lambda}=3 c^{2} M_{p}^{2} L^{-2}
$$

in which $3 c^{2}$ is a numerical constant. It is interesting to note that if the length scale $L$ is characterized by the size of the universe, the Hubble scale $H^{-1}$, then equation (3) gives a vacuum energy density of the right order of magnitude consistent with observations [13]. It is however pointed out that this yields a wrong equation of state parameter for dark energy, and other possible values for $L$ should be chosen such as the size of the future event horizon [14]. This conclusion is, however, based on the assumption of an independent evolution of energy densities of dark energy and dark matter. It is shown [15] that, if there is any interaction between these two components the identification of $L$ with $H^{-1}$ is possible and the equation of state parameter indicates late time acceleration. In a recent work [16], an interacting holographic dark energy is studied in Brans-Dicke theory. It is shown that in this framework there 
is a noninteracting limit and for a given set of parameters the equation of state parameter can be negative. Here we would like to generalize this work to the Brans-Dicke theory with a self-interacting potential. We shall assume that the matter contained in the universe consists of cold dark matter and an interacting holographic dark energy. We show that the potential term improves the behavior of the set of parameters. In particular, for a zero potential term the late time acceleration constrains the evolution of the gravitational coupling in the BransDicke theory in such a way that it is only allowed to increase with expansion. It is certainly unnatural if dynamical evolution of the scalar field would lead to this undesirable situation of going through an infinitely strong gravitational effect. We shall show that this behavior is improved for a nonzero potential.

This paper is organized as follows: In section 2, we first consider late time acceleration based on holographic principle in a generalized scalar-tensor theory. We assume that the matter contained in the universe consists of cold dark matter and holographic dark energy. Here these two types of matter are not conserved separately due to an interaction. As special cases, we then consider the limits of the model in general relativity and Brans-Dicke theory with potential. In the first parametrization (general relativity), we show that there is a non-interacting limit in the spatially curved Robertson-Walker spacetime. In the Brans-Dicke parametrization, we specifically study the effect of the potential of the scalar field in the behavior of equation of state parameter and the deceleration parameter. In section 3, we offer some concluding remarks.

\section{The Model}

We consider a model in which gravity is described by a scalar-tensor theory. The most general action functional for these theories is given by ${ }^{\dagger}$

$$
S=\frac{1}{2} \int d^{4} x \sqrt{-g}\left\{F(\phi) R+U(\phi) g^{\alpha \beta} \nabla_{\alpha} \phi \nabla_{\beta} \phi+V(\phi)\right\}+S_{m}\left(g_{\mu \nu}\right)
$$

where $S_{m}\left(g_{\mu \nu}\right)$ is the matter field action, $g$ is the determinant of the metric $g_{\mu \nu}, R$ is the Ricci scalar and the functions $F(\phi), U(\phi)$ and $V(\phi)$ are arbitrary functions of the real scalar field $\phi$. The only constraint on these functions is that $F(\phi)>0$ ensuring that graviton carries positive energy [17]. We also note that the matter action does not involve $\phi$ which means that the whole theory respects the weak equivalence principle [18].

Variations with respect to $g_{\mu \nu}$ and $\phi$ gives the field equations

$$
\begin{gathered}
F(\phi) G_{\mu \nu}=\left(T_{\mu \nu}+T_{\mu \nu}^{\phi}\right) \\
2 U(\phi) \square \phi+2 \nabla_{\gamma} U(\phi) \nabla^{\gamma} \phi-\frac{d U}{d \phi} \nabla_{\gamma} \phi \nabla^{\gamma} \phi+\frac{d F}{d \phi} R-\frac{d V}{d \phi}=0
\end{gathered}
$$

\footnotetext{
†Our sign convention is $(-+++)$ and we work in units in which $\hbar=c=1$.
} 
where

$$
\begin{gathered}
T_{\mu \nu}^{\phi}=U(\phi)\left(\nabla_{\mu} \phi \nabla_{\nu} \phi-\frac{1}{2} g_{\mu \nu} \nabla^{\alpha} \phi \nabla_{\alpha} \phi\right)+\left(\nabla_{\mu} \nabla_{\nu}-g_{\mu \nu} \square\right) F(\phi)-\frac{1}{2} g_{\mu \nu} V(\phi) \\
T_{\mu \nu}=-\frac{2}{\sqrt{-g}} \frac{\delta S_{m}}{\delta g^{\mu \nu}}
\end{gathered}
$$

In a cosmological context, we take $T_{\mu \nu}$ to consists of two interacting components, a pressureless dark matter and a holographic dark energy. Since both components do not evolve independently, a source (or loss) term must enter their energy balances

$$
\begin{gathered}
\dot{\rho_{m}}+3 H \rho_{m}=Q \\
\dot{\rho}_{\Lambda}+3 H\left(1+\omega_{\Lambda}\right) \rho_{\Lambda}=-Q
\end{gathered}
$$

where $\rho_{m}$ is energy density of matter and $\omega_{\Lambda}=\frac{p_{\Lambda}}{\rho_{\Lambda}}$ is equation of state parameter of dark energy. Following [15] we take the interaction term $Q$ as a decay process $Q=\Gamma \rho_{\Lambda}$ with $\Gamma$ being an arbitrary decay rate. If $\Gamma>0$, dark energy decays into the dark matter. Here we do not concern with the details of this decay process and do not answer the question that where exactly the dark energy is going to, see for instance [19] and references therein.

We specialize to Friedman-Robertson-Walker spacetime which is given by

$$
d s^{2}=-d t^{2}+a^{2}(t)\left\{\frac{d r^{2}}{1-k r^{2}}+r^{2}\left(d \theta^{2}+\sin ^{2} d \varphi^{2}\right)\right\}
$$

in which $a(t)$ is the scale factor and the spatial curvature $k=0,-1,1$ corresponds to flat, open and closed universes. For this metric and the aforementioned matter and energy components, the equations (5) can be written as

$$
\begin{gathered}
3 F\left(H^{2}+\frac{k}{a^{2}}\right)=\left(\rho_{m}+\rho_{\Lambda}\right)+\frac{1}{2} U \dot{\phi}^{2}-3 H \dot{F}+\frac{1}{2} V \\
F\left(3 H^{2}+2 \dot{H}+\frac{k}{a^{2}}\right)=-p_{\Lambda}-\frac{1}{2} U \dot{\phi}^{2}-\ddot{F}-3 H \dot{F}+\frac{1}{2} V
\end{gathered}
$$

where $H$ is the Hubble parameter defined by $H \equiv \frac{\dot{a}}{a}$. Note that the field equation of $\phi$ is not independent of equation (5) and the energy balances (9) and (10).

\subsection{The spatially flat case}

We intend now to obtain the equation of state parameter $\omega_{\Lambda}$ for a spatially flat spacetime, $k=0$. We first choose the Hubble horizon as the IR cutoff, i.e., $L=H^{-1}$. In this case the relation (3) takes the form

$$
\rho_{\Lambda}=3 c^{2} M_{p}^{2} H^{2}
$$

For mathematical convenience, we shall assume that $F(\phi)=\phi^{\alpha}$ in which $\alpha$ is a constant parameter. Following [16] and [20], we then restrict our analysis to the class of solutions for which the scalar field evolves as a power law of the scale factor $\phi \propto a^{n}$ with $n$ being a constant ${ }^{\ddagger}$.

\footnotetext{
${ }^{\ddagger}$ In principle, there is no physical reason for this choice and we find it mathematically convenient.
} 
Now, by combining these expressions for $\phi$ and $F(\phi)$ with equations (12), (13) and (14) and using (9), we obtain

$$
\omega_{\Lambda}=(1+r) \frac{(\alpha n+2) \frac{\Gamma}{H}-\alpha n(2 \alpha n+3)+V H^{-2} \phi^{-\alpha}-n^{2} U \phi^{-\alpha+2}}{3[\alpha n-(\alpha n+2) r]-n^{2} U \phi^{-\alpha+2}-V H^{-2} \phi^{-\alpha}}
$$

where $r \equiv \frac{\rho_{m}}{\rho_{\Lambda}}$. This relation clearly implies that $\omega_{\Lambda}$ is not necessarily a constant and can take negative values. The latter only sets some bounds on the numerical values of $\alpha, n$ and also possible dependence of $U$ and $V$ on $\phi^{\S}$. Before making a closer look at (15), let us write the deceleration parameter $q=-\left(1+\frac{\dot{H}}{H^{2}}\right)$. To this end, we combine (13) and (14) which gives

$$
q=\frac{1}{(\alpha n+2)}\left[1+\alpha n(\alpha n+2)+\left(3 c^{2} M_{p}^{2} \omega_{\Lambda}+\frac{1}{2} n^{2} U \phi^{2}-\frac{1}{2} V H^{-2}\right) \phi^{-\alpha}\right]
$$

Note that $q$ depends on $\Gamma$ and $r$ through the equation of state parameter $\omega_{\Lambda}$. To investigate the role of the functions $U(\phi)$ and $V(\phi)$ on the evolution of the parameters $\omega_{\Lambda}$ and $q$, we consider two special cases :

1) when $n$ is zero the scalar field becomes trivial and takes a constant configuration. In this case if we set $V=0$ and $F=(8 \pi G)^{-1}$, the action (4) reduces to the Einstein-Hilbert action. Thus, this is the limit of the model to general relativity. At this limit, the equation (15) reduces to

$$
\omega_{\Lambda}=-\left(1+\frac{1}{r}\right) \frac{\Gamma}{3 H}
$$

which is the result obtained in [15]. When there is no interaction, namely $\Gamma=0$, then $\omega_{\Lambda}=0$ and there is no late time acceleration. This dustlike equation of state was the basic problem that leads $\mathrm{Li}$ [14] to take the future event horizon rather than the Hubble radius as the IR cutoff. Moreover, the deceleration parameter (16) also reduces to

$$
q=\frac{1}{2}\left[1-3 c^{2} M_{p}^{2}\left(1+\frac{1}{r}\right) \frac{\Gamma}{3 H}\right]
$$

in which we have used (17). Thus, $q=\frac{1}{2}>0$ when $\Gamma=0$ and, as previously stated, there is no non-interacting limit.

2) the parametrization $\alpha=1$ and $U(\phi)=\frac{\omega}{\phi}$ corresponds to the Brans-Dicke model with a scalar field potential. In this case, the relation (15) reduces to

$$
\omega_{\Lambda}=(1+r) \frac{(n+2) \frac{\Gamma}{H}-(2 n+3) n-n^{2} \omega+V(\phi) H^{-2} \phi^{-1}}{3[n-(n+2) r]-n^{2} \omega-V(\phi) H^{-2} \phi^{-1}}
$$

This implies that $\omega_{\Lambda}$ can take negative values when

$$
\begin{gathered}
(n+2) \frac{\Gamma}{H}+\frac{V(\phi)}{\phi H^{2}}>(2 n+3) n+n^{2} \omega \\
3(n+2) r+n^{2} \omega+\frac{V(\phi)}{\phi H^{2}}>3 n
\end{gathered}
$$

\footnotetext{
$\S$ It should be pointed out that every couple $(\alpha, n)$ does not correspond to a real solution in this model. Moreover, one should in general consider $n(\alpha)$. See, for example, [21].
} 
or the reversed direction for the both inequalities. These conditions clearly depend on numerical values of $\Gamma, \omega$ and $n$ as well as the potential $V(\phi)$. When $V(\phi)=0$, the above inequalities set constraints on the decay rate of the dark energy into the dark matter. For a nonzero potential, on the other hand, we can consider more solutions of the field equations in which the set of parameters satisfies the inequalities (20) and (21). For instance, for a quartic potential [22] $V(\phi) \sim \phi^{4}$, the potential term $\frac{V(\phi)}{\phi H^{2}}$ increases with time so that at late times it dominates the conditions (20) and (21)

In this parametrization, deceleration parameter (16) reduces to

$$
q=\frac{1}{(n+2)}\left\{1+n(n+2)+\frac{1}{2} n^{2} \omega+3 c^{2} M_{p}^{2} \omega_{\Lambda} \phi^{-1}-\frac{1}{2} V(\phi) H^{-2} \phi^{-1}\right\}
$$

The signature flip of the deceleration parameter from positive to negative values depends crucially on the evolution of the negative term in (22). At the first glance, it seems to be important that whether the scalar field increases or decreases with time, namely that $n>0$ or $n<0$ respectively. However, the both cases may actually happen if the potential function in the last term on the right hand side of (22) has an appropriate functional form. In fact, for a suitably chosen potential this term may be set to increase with cosmic time regardless of the sign of $n$. For instance, for $n>0$ a power law [22] and for $n<0$ an inverse power law [24] potentials make the negative term of $(22)$ be an increasing function of time. In this case, at early times the potential term is negligible and the deceleration parameter is effectively positive whereas at late times it grows and dominates so that $q$ changes its sign.

The fact that the both cases $n>0$ and $n<0$ may fit the deceleration parameter with observations, is an improvement with respect to the case of zero potential function. In that case, there is no transition from deceleration to acceleration phase unless $n<0$ or $\phi$ being a decreasing function of time [16].

\subsection{The spatially curved cases}

Although it is a general belief that the universe is spatially flat, the spatial curvature may still have contribution to the field equations if the number of e-foldings is not very large. In fact, recent observations [23] favor the spatial curvature and imply that the effect of the latter, though much smaller than other energy components, can not be completely ruled out. For $k \neq 0$, the equations (15) and (16) generalize to

$$
\begin{gathered}
\omega_{\Lambda}=(1+r) \frac{(\alpha n+2) \frac{\Gamma}{H}-\alpha n(2 \alpha n+3)+V H^{-2} \phi^{-\alpha}-n^{2} U \phi^{-\alpha+2}-2 \Omega_{k}}{3[\alpha n-(\alpha n+2) r]-n^{2} U \phi^{-\alpha+2}-V H^{-2} \phi^{-\alpha}+6 \Omega_{k}} \\
q=\frac{1}{(\alpha n+2)}\left[1+\alpha n(\alpha n+2)+\Omega_{k}+\left(3 c^{2} M_{p}^{2} \omega_{\Lambda}+\frac{1}{2} n^{2} U \phi^{2}-\frac{1}{2} V H^{-2}\right) \phi^{-\alpha}\right]
\end{gathered}
$$

where $\Omega_{k}=\frac{k}{a^{2} H^{2}}$. To explore the consequences of these equations, let us write them in the two special cases considered above. Firstly, in the limit to general relativity, (23) and (24) reduce

IWe assume that $n>0$. For $n<0$, one may consider an inverse power law potentials [24] 
to

$$
\begin{gathered}
\omega_{\Lambda}=-\frac{1}{3}(1+r) \frac{\frac{\Gamma}{H}-\Omega_{k}}{r-\Omega_{k}} \\
q=\frac{1}{2}\left[1+\Omega_{k}+3 c^{2} M_{p}^{2} \omega_{\Lambda}\right]
\end{gathered}
$$

For $k=0$, these equations reduce to (17) and (18). Inspection of (25) reveals that for a closed universe $\left(\Omega_{k}>0\right)$ additional conditions should be satisfied with respect to the flat case. Specifically, the conditions $\frac{\Gamma}{H}>\Omega_{k}$ and $r>\Omega_{k}$, or the reversed direction of the inequality for the both, must hold in order that $\omega_{\Lambda}$ remains negative. It is interesting to note that contrary to the flat case, the relation (25) allows a non-interacting limit. It means that for an open universe $\left(\Omega_{k}<0\right), \Gamma=0$ still gives a consistent result. For a closed universe, the same is true if $r<\Omega_{k}$.

Secondly, in the Brans-Dicke parametrization, (19) and (22) generalize to

$$
\begin{gathered}
\omega_{\Lambda}=(1+r) \frac{(n+2) \frac{\Gamma}{H}-(2 n+3) n-n^{2} \omega+V(\phi) H^{-2} \phi^{-1}-2 \Omega_{k}}{3[n-(n+2) r]-n^{2} \omega-V(\phi) H^{-2} \phi^{-1}-6 \Omega_{k}} \\
q=\frac{1}{2}\left\{1+n(n+2)+\frac{1}{2} n^{2} \omega+\Omega_{k}+3 c^{2} M_{p}^{2} \omega_{\Lambda} \phi^{-1}-\frac{1}{2} V(\phi) H^{-2} \phi^{-1}\right\}
\end{gathered}
$$

It seems that introducing the curvature density in (27) does not alter seriously our qualitative picture of the equation of state parameter. However, we should set further conditions to have $\omega_{\Lambda}<0$. We should have $(n+2) \frac{\Gamma}{H}+\frac{V(\phi)}{\phi H^{2}}>(2 n+3) n+n^{2} \omega+2 \Omega_{k}$ together with $3(n+2) r+n^{2} \omega+\frac{V(\phi)}{\phi H^{2}}+6 \Omega_{k}>3 n$ or the reversed direction for the both inequalities. In these conditions $\Omega_{k}=+1$ or -1 for a closed and open universe, respectively. Inspection of the deceleration parameter in both cases reveals that the curvature density only affect the time of onset of the acceleration. For a closed universe the acceleration phase is delayed with respect to the flat case while for an open universe it starts in an earlier time.

\section{Conclusions}

We generalized holographic dark energy model to scalar-tensor theories of gravity. In our model, matter part consists of dark matter which is assumed to interact with the holographic dark energy. To explore the parameters $\omega_{\Lambda}$ and $q$, we restrict our attention to a specific functional form of $F(\phi)$, namely $F(\phi) \propto \phi^{\alpha}$. We have also assumed that the evolution of the scalar field is related to that of the scale factor in such a way as $\phi \propto a^{n}$. It is then shown that the desired behavior for $\omega_{\Lambda}$ and $q$ is possible for a wide range of the functions $U(\phi)$ and $V(\phi)$. Specifically, we have considered two special cases. In the limit of the model to general relativity the results of [15] recovered in the flat case. In the case of non-flat spaces, however, we have shown that $\Omega_{k}$ gives possibility to $\omega_{\Lambda}$ to take negative values even for $\Gamma=0$ for both closed and open universes.

We have also considered the limit to the Brans-Dicke model with a self-interacting scalar field. Although it is shown that for $V(\phi)=0$ the equation of state parameter may have negative values and $q$ can have a signature flip both in flat and curved spaces, the existence 
of the potential function improves the behavior of these parameters. Specifically, it should be emphasized that the deceleration parameter can change its sign during cosmic evolution for both $n>0$ and $n<0$, whereas in the case of zero potential it is only possible for $n<0$ [16]. It is shown that holographic energy is not compatible with phantom energy [25]. Thus we must impose $\omega \geq-1$. Combining this requirement with (27), results in

$$
r \geq \frac{2 n^{2}(\omega+1)-(n+2) \frac{\Gamma}{H}+8 \Omega_{k}}{(n+2) \frac{\Gamma}{H}+V(\phi) H^{-2} \phi^{-1}-n^{2} \omega-2\left(n^{2}+3 n+3\right)-2 \Omega_{k}}
$$

This constraint can be satisfied, for instance, for a quartic potential at late times.

\section{Acknowledgment}

The author would like to thank anonymous referees for their useful comments. This work is supported by the Office of Scientific Research of Shahid Rajaee University under the contract No.38227-7. 


\section{References}

[1] A. G. Riess et al., Astron. J. 116, 1009 (1998)

S. Perlmutter et al., Bull. Am. Astron. Soc., 29, 1351, (1997)

S. Perlmutter et al., Astrophys. J., 517, 565, (1997)

[2] L. Melchiorri et al., Astrophys. J. Letts., 536, L63, (2000)

S. Hanany et al., Astrophys. J. Letts., 545, L5, (2000)

C. B. Netterfield et al., Asrtrophys. J., 571, 604, (2002)

N. W. Halverson et al., Astrophys. J., 568, 38, (2002)

[3] D. N. Spergel et al., Astrophys. J. Suppl. 148, 175, (2003)

[4] S. Weinberg, Rev. Mod. Phys. 61, 1 (1989)

[5] A. G. Riess, Astrophys. J. 560, 49, (2001)

[6] B. Ratra and P. J. E. Peebles, Phys. Rev. D 37, 3406, (1988)

R. R. Caldwell, R. Dave and P. J. Steinhardt, Phys. Rev. Letts. 80, 1582, (1998)

P. J. Steinhardt, L. M. Wang and I. Zlatev, Phys. Rev. D 59, 123504, (1999)

A. D. Macorra and G. Piccinelli, Phys. Rev. D 61, 123503, (2000)

P. F. Gonzalez-Diaz, Phys. Rev. D 62, 023513, (2000)

S. A. Bludman and M. Roos, Phys. Rev. D 65, 043503, (2002)

[7] C. Armendariz-Picon, V. Mukhanov and P. J. Steinhardt, Phys. Rev. D 63, 103501, (2001)

T. Chiba, Phys. Rev. D 66, 063514, (2002)

R. J. Scherrer, Phys. Rev. Letts. 93, 011301, (2003)

[8] T. Padmanabhan, Phys. Rev. D 66, 021301, (2002)

A. Frolov, L. Kafman and A. Starobinsky, Phys. Letts. B 545, 8, (2002)

G. W. Gibbons, Phys. Letts. B 537, 1, (2002)

G. W. Gibbons, Class. Quan. Grav. 20, 321, (2003)

[9] R. R. Caldwell, Phys. Letts. B 545, 23, (2002)

J. Hao and X. Li, Phys. Rev. D68, 043501, (2003)

S. Nojiri and S. D. Odintsov, Phys. Letts. B 562, 147, (2003)

S. Nojiri and S. D. Odintsov, Phys. Letts. B 565, 1, (2003) 
[10] B. Feng, X. L. Wang and X. M. Zhang, Phys. Letts. B 607, 35, (2005)

Z. K. Guo, Y. S. Piao, X. M. Zhang and Y. Z. Zhang, Phys. Letts. B 608, 177, (2005)

[11] G. 't Hooft, gr-qc/9310026

L. Susskind, J. Math. Phys. 36, 6377, (1995)

[12] J. D. Bekstein, Phys. Rev. D 7, 2333, (1973)

J. D. Bekstein, Phys. Rev. D 9, 3292, (1974)

J. D. Bekstein, Phys. Rev. D 23, 287, (1981)

J. D. Bekstein, Phys. Rev. D 49, 1912, (1994)

[13] A. G. Cohen, D. B. Kaplan and A. E. Nelson, Phys. Rev. Letts. 82, 4971, (1999)

[14] H. D. S. Hsu, Phys. Letts. B 594, 13, (2004)

M. Li, Phys. Letts. B 603, 1, (2004)

[15] D. Pavon and W. Zimdahl, Phys. Letts. B 628, 206 (2005)

[16] N. Banerjee and D. Pavon, Phys. Letts. B 647, 447, (2007)

[17] T. Damour and G. Esposito-Farese, Class. Quantum. Grav. 9, 2093, (1992)

B. Boisseau, G. Esposito-Farese, D. Polarski and A. A. Starobinsky, Phys. Rev. Lett. 85, $2236,(2000)$

[18] C. M. Will, Theory and Experiments in Gravitational Physics (Cambridge University Press, Cambridge, England, 1993)

E. Poisson and C. M. Will, Phys. Rev. D 52, 848, (1995)

[19] J. S. Alcaniz, Braz. J. Phys. 36, 1109, (2006)

[20] M. R. Setare, Phys. Letts. B 644, 99, (2007)

[21] S. Carloni, J. A. Leach, S. Capozziello and P. K. S. Dunsby, Class. Quantum Grav. 25, 035008, (2008). 
[22] J. Frieman, C. T. Hill, A. Stebbins and I. Waga, Phys. Rev. Lett. 75, 2077, (1995)

[23] C. L. Bennett et al., Astrophys. J. Suppl. 148, 1, (2003)

D. N. Spergel, Astrophys. J. Suppl. 148, 175, (2003)

A. G. Riess et al., Astron. J. 607, 665 (2004)

M. K. Mak and T. Harko, Europhys. Lett. 60, 155, (2002)

[24] P. J. E. Peebles and B. Ratra, Astrophys. J. Lett. 325, L17, (1988)

B. Ratra and P. J. Peebles, Phys. Rev. D 37, 3406, (1988)

[25] D. Bak and S. J. Rey, Class. Quantum Grav. 17, L83 (2000)

E. E. Flanagan, D. Marolf and R. M. Wald, Phys. Rev. D 62084035 (2000) 